\title{
Administración de servicios de alimentación a colectividades desde el enfoque del nutriólogo
}

\section{Administration of food services to communities from the nutritionist's approach}

\author{
Ilse H. Ortega Ibarra ${ }^{a}$, Edú Ortega Ibarra ${ }^{b}$, Edna Rodríguez-López ${ }^{c}$
}

\begin{abstract}
:
Administrative sciences have a specific focus on food, since they approach food from different steps, logistical and regulatory ramifications. Thus, food is seen as a systematic process that always must be in balance. Food service is carried out by professionals with specific and general approaches (from administrative and social sciences, food technology, law and public health), who always seek to reduce health risks and secure that food achieves its nutritional objective. In this sense, the nutrition professional is in charge both of preserving the health of diners, as well as management activities, implementing good manufacturing practices, quality standards and food safety and processes involved in planning, organization, direction, administration, evaluation and control of activities and human resources. Moreover, the nutrition professional is always in a constant process of research and innovation in order to improve their service continuously. Food service management in communities is monitored by global and local organizations to control food safety. In Mexico, the responsible monitoring institutions are the Ministry of Health, the Ministry of Agriculture, Livestock, Rural Development, Fisheries and Food, among other institutions. The relationship between quality and food safety is crucial, since is essential to prevent food-borne diseases, which currently represent a public health problem in Mexico.
\end{abstract}

\section{Keywords:}

Food services, food management, food distribution, food higiene, food security

\section{Resumen:}

Las ciencias administrativas tienen un enfoque específico sobre la alimentación, ya que la abordan desde distintos pasos, ramificaciones logísticas y normativas; por lo que la alimentación es vista como un proceso sistemático que siempre debe estar en equilibrio. El servicio de alimentación es llevado a cabo por profesionales con enfoques específicos y generales (desde las ciencias administrativas, sociales, tecnología de alimentos, derecho y salud pública), siempre buscando reducir los riesgos sanitarios y que los alimentos cumplan con su objetivo nutricional. En este sentido, el profesional de la nutrición se encarga tanto de preservar la salud de los comensales como de actividades de gestión, implementando buenas prácticas de manufactura, normas de calidad e inocuidad alimentaria; así como de los procesos involucrados en la planificación, organización, dirección, administración, evaluación y control tanto de las actividades como del recurso humano. Además, el profesional de la nutrición se encuentra siempre en un proceso constante de investigación e innovación con el objetivo de mejorar su servicio de manera continua. La administración de servicios de alimentación a colectividades está vigilada por diversos organismos a nivel mundial y local con el fin de controlar la inocuidad alimentaria. En México, los encargados son la Secretaría de Salud (SSA) y la Secretaría de Agricultura, Ganadería, Desarrollo Rural, Pesca y Alimentación (SAGARPA), entre otras dependencias. La relación entre calidad e inocuidad alimentaria es de suma importancia es indispensable para prevenir las enfermedades trasmitidas por los alimentos, que en la actualidad representan un problema de salud pública en nuestro país.

\footnotetext{
Universidad del Istmo, campus Juchitán de Zaragoza, Oaxaca., https://orcid.org/0000-0002-1104-2949, Email: ihoi@bizendaa.unistmo.edu.mx

b Autor de Correspondencia, Universidad del Istmo, campus Juchitán de Zaragoza, Oaxaca. E.O.I.: https://orcid.org/0000-0002-6504-7366, Email eoi@bizendaa.unistmo.edu.mx

Universidad del Istmo, campus Juchitán de Zaragoza, Oaxaca. E.R.-L. https://orcid.org/0000-0001-6688-8362, Email:
} eirl@bizendaa.unistmo.edu.mx 


\section{Palabras Clave:}

Servicios de alimentación, administración de alimentos, seguridad alimentaria, distribución de alimentos, higiene de los alimentos, nutriólogo

\section{Introducción}

La alimentación y la nutrición humana son términos que se estudian desde diversas miradas, tanto que se considera como una ciencia que involucra a la tecnología, fomentando la creación de nuevas teorías. Actualmente el proceso definido como "alimentación" puede ser estudiado desde las ciencias administrativas, donde se le otorga un enfoque necesario para que cumpla la función, esto mediante procesos, ramificaciones logísticas y cumpliendo con normativas; alimentarse llega a verse desde una postura sistemática, que, al interrumpirse algún eslabón, puede generar un gran desequilibrio y no cumplir la meta. Los procesos administrativos de organización y operativos en instituciones que otorgan un servicio de alimentación, deben ser focalmente vistos y regidos por quienes acudan a él, tanto así que se entiende que este tipo de servicio contiene un trasfondo teórico y práctico con el fin de asegurar que el alimento llegue en condiciones idóneas a la persona.

Un Servicio de Alimentación (SA) es intervenido por diversas personas con enfoques específicos y generales, los cuales buscan un fin común tratar de reducir los riesgos sanitarios. El proceso de un SA debe ser estudiado desde un enfoque de las ciencias administrativas, sociales, tecnología de alimentos, derecho y en salud pública. Un SA es visto de manera internacional y nacional como una actividad que provee acciones seguras a quien lo aborde, es así como cualquier grupo etario sea visto como un mismo ente que requiere tener la seguridad que lo que ingiere cubre con un proceso estrictamente vigilado y que, además, cumple con el objetivo de ser nutritivo.

El profesional de la nutrición tiene un reto en los servicios de alimentación ya que se encarga tanto de preservar la salud de los comensales como de actividades de gestión implementando buenas prácticas de manufactura, además de efectuar normas de calidad e inocuidad alimentaria, así como planificar, organizar, dirigir, administrar, evaluar y controlar actividades, recurso humano y procesos así como la necesidad de investigar e innovar todo lo antes hecho con el objetivo de mejorar de modo continuo.

Estudiar esta área de la nutriología llega a ser complicado, ya que la evidencia científica debe ser analizada, por aquellos recursos humanos en salud que estén estudiándola, ya que se nota que existe una necesidad laboral de tener nutriólogas y nutriólogos con los conocimientos mínimos, debido a que la oferta laboral refiere un SA en instituciones públicas o particulares de una manera recurrente y en diversas partes del país. Por lo que, estudiar las generalidades, los elementos básicos mínimos requeridos del área mencionada es necesaria y urgente en cubrir; independientemente si son servicios de alimentos comerciales (comedores industriales privados, restaurantes, empresas particulares, hospitales particulares, entre otros) y los no comerciales (hospitales, escuelas públicas, centros públicos).

\section{Antecedentes internacionales en normativas internacionales}

Se conoce que leyes y normas incluyen actividades para delimitar especificaciones para servicios, productos, algunos procesos, desde una necesaria evaluación. En cuanto a las normativas, reflejada en reglamentos ante características, cantidad, calidad, todo para garantizar que la producción y comercialización sea accesible, de fácil camino; pero sobre todo confiables y acercarse a una de las áreas de la seguridad alimentaria y nutricional, como lo es la utilización biológica alimentaria (que se acerca a la inocuidad), para reducir riesgos sanitarios y primordialmente otorgarle al comensal, usuario o paciente esa seguridad que lo que ingiere está en óptimas condiciones, respetadas por lineamientos definidos por la evidencia científica.

Con el fin de evitar fraudes en productos alimenticios, fue que la legislación en alimentos surgió en diversos países, en donde se justificaban que eran productos con posibles efectos negativos, agudos y crónicos en cualquier sistema del cuerpo humano, en sí inclinando a una irregularidad en el equilibrio del ser humano, esto desde la mirada de la Salud Pública Global, tomando en cuenta variables que para ese entonces eran vistos como contaminantes químicos, físicos y biológicos, esto de acuerdo a experiencias y vivencias. Estando el mundo viviendo por irregularidades sanitarias, y de paso por alimentos nuevos y sin vigilancia, fue que, en el siglo $X X$, surgió la necesidad de fijar normas sanitarias en materia de alimentación internacional, encaminado a que los alimentos procesados 0 bien los naturales, se vean controlados por vigilantes con lineamientos que coincidiesen en reglas y acuerdos, para una adecuada 
comercialización y consumo por visitantes internacionales. ${ }^{1}$

Tomando en cuenta estándares y normas internacionales, fue que la Federación Internacional de Lechería creó protocolos, lineamientos y fijó reglas para aplicarse en leche y sus varios derivados; para inicios de la segunda mitad del siglo XX, la Organización para la Alimentación y la Agricultura (FAO) por sus siglas en inglés y la Organización Mundial de la Salud (OMS) trabajaron en conjunto para estudiar, elaborar y trabajar en cuestión de un tópico nuevo, innovador para ese entonces, como lo que hoy conocemos como "higiene e inocuidad de alimentos", esto desde un punto de vista internacional. Es tanto que, actualmente son líderes en procesos normativos, asesorando con evidencia científica a las diversas naciones en esa área; principalmente para prevenir y evitar casos peligrosos por contaminantes físicos, químicos y/o biológicos y sumando los procesos, métodos y técnicas relacionados en la alimentación a colectividades. ${ }^{1,2}$

El Codex Alimentarius (CA). Es un organismo surgió en 1963 siendo parte de la alianza entre la OMS y la FAO, fijando nuevas responsabilidades específicas para las nuevas naciones que se integraban y con el surgimiento de acuerdos entre países y continentes en materia de intercambio de bienes y servicios de alimentación. EI CA para ese entonces, delimitó nuevas reglas, acuerdos, códigos de prácticas comerciales, con el objetivo de salvaguardar la salud de consumidores y propiciar la adecuada práctica comercial a nivel global; entre sus principios identifica puntos esenciales, como es la higiene de alimentos tomando en cuenta la "cadena alimentaria" desde la producción primaria hasta que está frente al comensal-consumidor final. Afortunadamente, promulgó normas que cubren aspectos primordiales para la trazabilidad y seguridad alimentaria global, como lo son "la composición, y calidad", antes y durante la distribución; reglamentando que deben estar bajo métodos y técnicas de análisis, aunado con un etiquetado de interpretación internacional, códigos de práctica para la producción, manipulación, distribución y transporte; evaluando a aquellos alimentos que se iniciaron con modificaciones genéticas. ${ }^{2,3}$

Para su creación, el Codex Alimentarius, siendo parte de dos grandes organismos; se asesoraron científicamente para establecer dosis máximas de referencia internacional y en particular para continentes, en cuanto a sustancias tóxicas, contaminantes, aditivos naturales y artificiales, limitaciones máximas en residuos (como en los plaguicidas y medicamentos-suplementos en animales para consumo humano). Dejando nuevas áreas de estudio como los "criterios microbiológicos" obligatorios para que los alimentos naturales y procesados sean evaluados previamente para ser consumidos en cualquier parte del mundo. Resguardar la seguridad alimentaria y nutricional global es tarea de esa triada de organismos, intencionalmente para promover proyectos, programas, investigaciones y capacitaciones para las naciones involucradas, tanto para que el Codex Alimentarius y los gobiernos consideren que es elemental para los diversos determinantes sociales en mejora de la salud global. ${ }^{2,4}$

\section{Breve conceptualización de los servicios de alimentación}

Izquierdo et al., mencionan que "la alimentación es una cadena de hechos que comienza en el cultivo, selección, preparación del alimento, hasta las formas de presentación y el consumo de un grupo de ellos", haciendo alusión de "eslabones" para que el tópico en cuestión sea entendido como un proceso para poder tener un resultado final ${ }^{5}$, lo ideal sería aunarle el manejo higiénico, pero para ello ya existen otras áreas de estudio. Entre tantas ciencias aunadas a la nutriología se encuentra la "administración", gran acompañante de la salud pública, tanto que tiene en sus múltiples áreas, la del Servicio de Alimentación a Colectividades; contemplando diversos temas que atender, fundamentados por los diversos organismos a nivel internacional y nacional. $^{6}$

Para los Servicios de Alimentación se deben considerar reglamentos de sitios, planeaciones estratégicas y de contingencia, generalidades de procesos, interpretación de análisis microbiológicos alimentarios, identificación de tipos de servicios al consumidor-comensal, planeación de menús, medición de satisfacción de quien ingiere, costos, gastos y control del almacén, compras y selección de proveedores, entre otros temas. Creación de organigramas de comedores comerciales y no comerciales, mapeos de procesos (gráficos simples y accesibles), diagramas de flujos, proyectos de mejora continua, menú cíclico y retroalimentación del sitio de práctica o del área fija ${ }^{7}$, adicionando que esto son subtemas que deben ser estudiados durante un tiempo considerado para que el nutriólogo fluya en el área, sin dejar a un lado los aprendizajes en materia de nutrición clínica, tecnología de alimentos y salud pública.

Por ello es de gran importancia la enseñanza de Servicios de Alimentación dentro de la formación del profesional de Nutrición ya que es fundamental que sean capaces de enfrentar los retos actuales y por lo tanto le permitan 
desarrollar competencias de liderazgo, dominio de procesos regulatorios y gubernamentales en el área, además del adecuado manejo de recursos entre otros aspectos que son necesarios en un servicio de alimentos. $^{8}$

Definiendo un "Servicio de Alimentación" (SA) se entiende como una "organización técnico-administrativa cuya función es entregar alimentación a grupos de personas" 9; otra definición es "una organización técnico administrativa de tipo directo en el mantenimiento y recuperación de la salud, con normas y procedimientos bien definidos que facilitan su función de planificar, elaborar y distribuir alimentos a colectividades" ${ }^{10,11}$ por otra parte se encuentra una descripción más amplia del concepto, "los servicios de alimentación son instalaciones donde preparan y sirven alimentos para el consumo humano. Los mismos son de carácter social (cantinas escolares, comedores escolares, universitarios, laborales, otros) o comercial (restaurantes, bufet y servicios de comida rápida). Para que un servicio de alimentación sea de calidad debe planificarse todo el proceso desde la recepción de los alimentos, preparación y conservación hasta su distribución, bajo estándares técnicos y sanitarios, donde se generen alimentos con alto valor nutricional, inocuidad y óptimas características sensoriales". 12

Entre las funciones que se delimitan a nivel nacional, se encuentra una propuesta por parte del gobierno del Estado de México, la cual enumera una lista de acciones para que cubra con lo que es un SA, iniciando con una "provisión y distribución de alimentos: suministro de insumos perecederos y no perecederos necesarios para la preparación de los alimentos" retomando ciertos temas por parte de Tejeda y colaboradores en 2007 y 2016, además de Mercado Zepeda y equipo (2018), quienes publicaron lo mínimo a atender en un servicio de ese tipo, otra acción es la "capacitación y orientación alimentaria: formación que deben recibir todos los actores involucrados en temas de: higiene personal, conservación de los alimentos; condiciones de almacenaje; tiempo para su consumo-caducidad", en tercera, una "supervisión y vigilancia: verificación del cumplimiento de los criterios nutrimentales; del manejo, preparación e higiene de alimentos...", después una "evaluación, la cual comprende la valoración periódica de las acciones implementadas por todos los actores e instancias que participan" y por último una "certificación, que asegura por escrito que el servicio o la capacitación recibida cumplen con los requisitos especificados" 7,10, 11 . Para un mejor entendimiento, Tejeda en 2006 propone un modelo de un Sistema de Servicio de Alimentación (Figura 1).
La vigilancia de la seguridad alimentaria y nutricional (SAN) y de la trazabilidad, tanto que tiene en uno de sus "Pilares", el de la Utilización Biológica Alimentaria donde se encuentra la higiene e inocuidad, visto como un reto para ciertos países en vías de desarrollo o subdesarrollados; revelando que influye en los datos de morbilidad y mortalidad asociadas por contaminación física, química y biológica alimentaria, además la SAN estudia la microbiología alimentaria, donde considera a la "calidad del agua para riego en la agricultura y ganadería" y a las "prácticas de higiene e inocuidad del manipulador de alimentos" como dos de los aspectos cumbres, críticos para generar un caos en la cadena de producción. Al tratar de garantizar que la cantidad y calidad de los alimentos, se considera una obligación por los diversos actores involucrados, desde la producción, comercialización y hasta que llega "al plato" o a las manos del consumidor-paciente-comensal. Es necesario que la capacitación sobre las Enfermedades Transmitidas por Alimentos sea recurrente y actualizada por parte de los manipuladores. ${ }^{13,14}$ Se sabe que los SA y las nuevas áreas de vigilancia, deben estar aliadas, actualizadas, y en alianza para la generación de nuevos conocimientos, estrategias y propuestas; ya que no relucen publicaciones científicas actualizadas en el país y que puedan aunar en beneficio de esta área que el nutriólogo está encontrando espacio laboral.

Sí bien existen algunas, son focalizadas principalmente en Servicios de Alimentación en niveles muy específicos de la salud pública, basadas en normativas específicas, como la de España o Portugal, que tienen alianzas legales para el intercambio de insumos, bajo estándares exclusivos, basados en el Codex Alimentarius y sus Ministerios de Salubridad. Para el caso de México, existen diversas Normas oficiales mexicanas $y$ publicaciones de algunas instituciones académicas, de investigación que son urgentes en adquirir o crear para hospitales, empresas, universidades, organismos, asociaciones civiles, organizaciones municipales, entre otros; tanto públicos como particulares. 
Modelo de un Sistema de Servicio de Alimentación

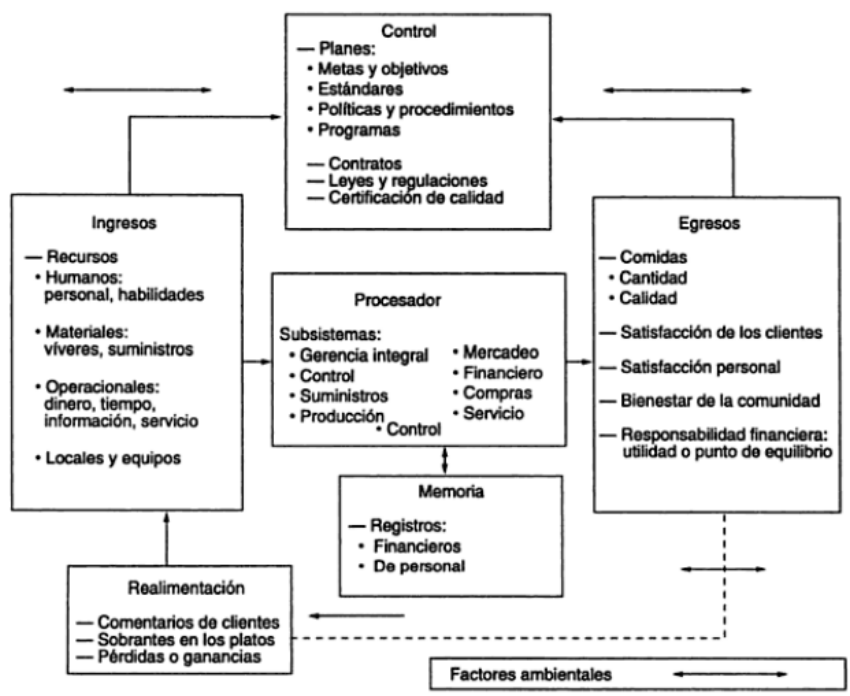

Figura 1. Modelo de un Sistema de Servicio de Alimentación ${ }^{11}$

\section{Normatividad oficial mexicana en salud alimentaria}

En México, existen 2 agencias principales que se encargan de la inocuidad de los alimentos frescos y procesados. Dichas agencias son responsabilidad de dos Secretarías de Estado: la Secretaría de Salud (SSA) y la Secretaría de Agricultura, Ganadería, Desarrollo Rural, Pesca y Alimentación (SAGARPA). Conforme a la Ley General de Salud, la SSA ejerce las atribuciones de regulación, control y fomento sanitario, a través de la Comisión Federal para la Protección contra Riesgos Sanitarios (COFEPRIS) y la SAGARPA se encarga de los aspectos de inocuidad a través del Servicio Nacional de Sanidad, Inocuidad y $\quad$ Calidad Agroalimentaria (SENASICA).

Existen lineamientos formales que marcan normas de carácter obligatorio y reglas para que el resultado cumpla con el objetivo; para ello, existen los procesos que incluyen recursos que se encargan de obtener resultados por medio de un método y/o técnica, cumpliendo por una vigilancia para ser eficiente e idealmente eficaz. Esto en cualquier área administrativa, en las ciencias de la salud, y en este caso en la administrativa en salud alimentaria; para México, se tiene la Ley Federal sobre Metrología y Normalización se encarga de promover la observancia y elaboración de Normas Oficiales Mexicanas (NOM), además de Normas Mexicanas (NMX); en donde se facilita la cadena de producción y comercialización, buscando la calidad del alimento; hasta mencionando que sea competitiva. ${ }^{15,16}$ Como bien menciona Helleriegel "dichas normas o reglas son lineamientos formales para el comportamiento de los empleados mientras están en el trabajo, las cuales pueden ayudar a proporcional la disciplina que necesita una organización para alcanzar las metas". ${ }^{17}$

Además, se debe considerar al Sistema Federal de Salud, la Comisión Federal para la Protección contra Riesgos Sanitarios (Cofepris) que para 2009, decretó la Norma Oficial Mexicana NOM-251-SSA1-2009: "Prácticas de higiene para el proceso de alimentos, bebidas o suplementos alimenticios", entrando en vigor un año después; en el cual se delimitan requisitos básicos, mínimos para buenas prácticas de higiene para vigilarse en el proceso de diversidad alimentaria que se comercializa con diversos fines, como lo son los alimentos procesados, ultra procesados, bebidas, suplementos, materias primas; todo para evitar contaminantes, siendo transparentes y asegurando al comensal que no hay riesgos sanitarios. ${ }^{18}$

Dentro de las normas que también regula un SA se encuentra la NOM-120-SSA1-1994: Prácticas de higiene y sanidad para el proceso de alimentos, bebidas no alcohólicas y alcohólicas, donde se establecen los requisitos para un adecuado procesamiento de los alimentos con el fin de reducir riesgos para la salud a la población consumidora. $^{19}$ La NOM-093-SSA1-1994: Prácticas de higiene y sanidad en la preparación de alimentos que se ofrecen en establecimientos fijos, establece acciones que deben efectuarse con el fin de contribuir a la protección de la salud del consumidor que permitan reducir aquellos factores que influyen durante su preparación en la transmisión de enfermedades por alimentos (ETA) ${ }^{20}$

Existiendo normas públicas y algunas particulares, como las que elabora la Sociedad Mexicana de Normalización y Certificación S.C NORMEX, que para 1993 sigue elaborando normas (NMX-NORMEX) y certificaciones, algunas verificaciones, evaluando procesos y sistemas, algunos productos, todo para dar una resolución al consumidor, que si es o no "de calidad" el servicio. Para las institucionales, se focaliza la NMX-F-605-NORMEX2016: Manejo higiénico en el servicio de alimentos preparados para la obtención del Distintivo "H", con fecha de actualización y aprobación como Norma Mexicana el 7 de diciembre de 2015 (NORMEX, 2016); derivada de la NMX-F-605-NORMEX-2004. La MNX-CC-9000-IMNC200 (norma ISO-9000: Sistemas de gestión de calidadFundamentos y vocabulario) considera a los procesos como necesarios para resultados. ${ }^{16}$ 
Coincide en ciertos puntos con las NOM, tal como que las organizaciones operen eficazmente, identificando $y$ gestionando procesos relacionados, definiendo esto como "enfoque basado en procesos" ${ }^{21}$, Martínez en 2005 propone una clasificación para dichos procesos, iniciando con: 1. "Procesos clave" (relación directa con la misión de la organización, ofrecidos a los clientes externos), 2. "Procesos de apoyo" (facilitan y hacen posible los procesos) y 3 . "Procesos de control" (valor de uso de los recursos que miden eficacia operativa y calidad). ${ }^{22}$

La Secretaría de Turismo y la Secretaría de Salud, bajo los lineamientos de la NMX-F-605-NORMEX-2016, proponen una estrategia para certificar establecimientos institucionales mientras presten un servicio de alimentación y bebidas; esto en beneficio de la seguridad alimentaria y nutricional desde la mirada de la microbiología alimentaria. Salvaguardando las buenas prácticas de higiene y sanidad, manipulación de alimentos. ${ }^{23}$ Ortega (2019) proporciona la tabla 1 para visualizar los elementos evaluadores, objetivos, aplicación y el nivel de impacto; es necesario que se estudie cada una de ellas, se entienda la génesis de la misma y que además se reconozcan los actores que lo crearon, ya que son piezas fundamentales ante el área de la Administración en Servicios a Colectividades, aunado que debe diferenciar lo que implica una NOM y una NMX. ${ }^{15}$

Tabla 1. Comparación de objetivo, aplicación y nivel de impacto de los elementos evaluadores

\begin{tabular}{|c|c|c|c|}
\hline $\begin{array}{l}\text { Element } \\
\text { os } \\
\text { evaluado } \\
\text { res. }\end{array}$ & Objetivo & Aplicación & $\begin{array}{l}\text { Nivel de } \\
\text { impacto }\end{array}$ \\
\hline $\begin{array}{l}\text { Norma } \\
\text { Oficial } \\
\text { Mexicana } \\
\text { NOM- } \\
251 \text { - } \\
\text { SSA1- } \\
2009 \text {, } \\
\text { "Prácticas } \\
\text { de } \\
\text { higiene } \\
\text { para el } \\
\text { proceso } \\
\text { de } \\
\text { alimentos } \\
\text {, bebidas } \\
\text { o } \\
\text { suplemen }\end{array}$ & $\begin{array}{l}\text { Establecer } \\
\text { requisitos } \\
\text { mínimos de } \\
\text { buenas } \\
\text { prácticas } \\
\text { de higiene } \\
\text { que deben } \\
\text { observarse } \\
\text { en el } \\
\text { procesami } \\
\text { ento de } \\
\text { alimentos, } \\
\text { bebidas, } \\
\text { suplement } \\
\text { os alimenticio } \\
\text { s y sus }\end{array}$ & $\begin{array}{l}\text { Todas } \\
\text { aquellas } \\
\text { organizacion } \\
\text { es } \\
\text { encargadas } \\
\text { del } \\
\text { suministro } \\
\text { alimentario y } \\
\text { de manera } \\
\text { obligatoria a } \\
\text { las personas } \\
\text { físicas o } \\
\text { morales que } \\
\text { se dedican al } \\
\text { proceso de } \\
\text { alimentos, } \\
\text { bebidas y }\end{array}$ & $\begin{array}{l}\text { Nacional. } \\
\text { Normas } \\
\text { Oficiales } \\
\text { Mexicanas } \\
\text { (lineamient } \\
\text { os de } \\
\text { observanci } \\
\text { a obligatoria) } \\
\text {. }\end{array}$ \\
\hline
\end{tabular}

\begin{tabular}{|c|c|c|c|}
\hline $\begin{array}{l}\text { tos } \\
\text { alimentici } \\
\text { os". }\end{array}$ & $\begin{array}{l}\text { materias } \\
\text { primas a fin } \\
\text { de evitar su } \\
\text { contaminac } \\
\text { ión, y por lo } \\
\text { tanto } \\
\text { mejorar la } \\
\text { transparen } \\
\text { cia y } \\
\text { seguridad } \\
\text { a lo largo } \\
\text { de la } \\
\text { cadena } \\
\text { alimentaria } \\
\text {. }\end{array}$ & $\begin{array}{l}\text { suplementos } \\
\text { alimenticios } \\
\text { dentro de } \\
\text { territorio } \\
\text { nacional } \\
\text { mexicano. }\end{array}$ & \\
\hline $\begin{array}{l}\text { NMX-F- } \\
605- \\
\text { NORMEX } \\
-2016 \\
\text { "Alimento } \\
\text { s - } \\
\text { manejo } \\
\text { higiénico } \\
\text { en el } \\
\text { servicio } \\
\text { de } \\
\text { alimentos } \\
\text { preparad } \\
\text { os para la } \\
\text { obtención } \\
\text { del } \\
\text { Distintivo } \\
\text { "H”". }\end{array}$ & $\begin{array}{l}\text { Promover y } \\
\text { fomentar } \\
\text { las buenas } \\
\text { prácticas } \\
\text { de higiene } \\
\text { y sanidad a } \\
\text { lo largo de } \\
\text { la cadena } \\
\text { productiva } \\
\text { primaria y } \\
\text { secundaria }\end{array}$ & $\begin{array}{l}\text { Todos } \\
\text { aquellos } \\
\text { establecimie } \\
\text { ntos de } \\
\text { hostelería y } \\
\text { restauración } \\
\text { que de } \\
\text { manera } \\
\text { voluntaria } \\
\text { desearan } \\
\text { mejorar sus } \\
\text { procesos } \\
\text { para } \\
\text { garantizar la } \\
\text { seguridad e } \\
\text { higiene de } \\
\text { los alimentos } \\
\text { incidiendo } \\
\text { significativa } \\
\text { mente en la } \\
\text { promoción } \\
\text { turística de } \\
\text { México ante } \\
\text { el mundo }\end{array}$ & $\begin{array}{l}\text { Nacional. } \\
\text { Expresan } \\
\text { una } \\
\text { recomenda } \\
\text { ción de } \\
\text { parámetros } \\
\text { o } \\
\text { procedimie } \\
\text { ntos. } \\
\text { Sí una } \\
\text { NOM } \\
\text { expresa la } \\
\text { obligatoried } \\
\text { ad de una } \\
\text { NMX, ésta } \\
\text { tendrá que } \\
\text { cumplirse. }\end{array}$ \\
\hline $\begin{array}{l}\text { Codex } \\
\text { Alimentari } \\
\text { us }\end{array}$ & $\begin{array}{l}\text { Garantizar } \\
\text { alimentos } \\
\text { inocuos y } \\
\text { de calidad } \\
\text { a todas las } \\
\text { personas y } \\
\text { en } \\
\text { cualquier } \\
\text { lugar, bajo } \\
\text { las normas } \\
\text { alimentaria } \\
\text { s, } \\
\text { directrices } \\
\text { y códigos }\end{array}$ & $\begin{array}{l}\text { Las normas } \\
\text { y textos } \\
\text { afines del } \\
\text { Codex no } \\
\text { sustituyen ni } \\
\text { son una } \\
\text { solución } \\
\text { alternativa a } \\
\text { la legislación } \\
\text { nacional. Las } \\
\text { leyes y } \\
\text { procedimient } \\
\text { os } \\
\text { administrativ }\end{array}$ & $\begin{array}{l}\text { Internacion } \\
\text { al. } \\
\text { Normas } \\
\text { alimentaria } \\
\text { s, } \\
\text { directrices } \\
\text { y códigos } \\
\text { de } \\
\text { prácticas } \\
\text { internacion } \\
\text { ales. }\end{array}$ \\
\hline
\end{tabular}




\begin{tabular}{|l|l|l|}
\hline de & os de cada \\
prácticas & país \\
internacion & contienen \\
ales. & $\begin{array}{l}\text { disposicione } \\
\text { s que es } \\
\text { necesario } \\
\text { cumplir. }\end{array}$ \\
& & \\
\hline
\end{tabular}

Fuente: Ortega Ibarra, E., \& Hernández Jiménez, $A$. (2019). Manejo higiénico en el servicio de alimentos: Codex Alimentarius y Normas Oficiales Mexicanas.

De igual manera, se tiene también al Distintivo "H", por parte de SECTUR y dentro de la NMX-F-605-NORMEX2016 con el fin de disminuir la incidencia de las Enfermedades de Transmisión Alimentaria, hace relucir puntos críticos y contrarrestarlos; tiene una vigencia de un año, de no evaluarse en su caducidad la institución puede ser sancionada interna y externamente por instituciones que ofrecen servicio de alimentación parcial o todo el día, quien vigila el cumplimiento es Cofepris (Tabla 2). Para verificar esta información está regida por una lista de verificación que se deberá cumplir en un $90 \%$ de satisfacción y que contempla los siguientes puntos: recepción de alimentos, almacenamiento, manejo de sustancias químicas, refrigeración y congelación, área de cocina, preparación de alimentos, área de servicio, agua, hielo, servicios sanitarios para empleados, entre otros. ${ }^{23}$

Tabla 2. Pasos para la obtención del Distintivo "H"

\begin{tabular}{|c|c|}
\hline $\begin{array}{lr}\text { Paso } & 1 \\
\text { Contratación } & \text { de } \\
\text { consultor "H" } & \end{array}$ & $\begin{array}{l}\text { Selección del consultor "H" } \\
\text { credencial vigente por } \\
\text { SECTUR. }\end{array}$ \\
\hline $\begin{array}{l}\text { Paso } \\
\text { Diagnóstico }\end{array}$ & $\begin{array}{l}\text { El consultor "H" realiza pre- } \\
\text { auditorias con la finalidad de } \\
\text { detectar puntos críticos, } \\
\text { requerimientos y puntos de mejora } \\
\text { que permita la implementación del } \\
\text { Sistema de Gestión "H" en las } \\
\text { áreas específicas de: recepción, } \\
\text { almacenamiento, preparación y } \\
\text { servicio. }\end{array}$ \\
\hline $\begin{array}{l}\text { Paso } \\
\text { Capacitación }\end{array}$ & $\begin{array}{l}\text { Capacitación en aula y campo } \\
\text { impartido por el consultor a todo el } \\
\text { personal operativo, mandos } \\
\text { medios y altos con el curso: } \\
\text { "Manejo Higiénico de los } \\
\text { Alimentos" con duración de } 10 \\
\text { horas. }\end{array}$ \\
\hline $\begin{array}{l}\text { Paso } 4 . \\
\text { Implementación }\end{array}$ & $\begin{array}{l}\text { Implementación del proceso de } \\
\text { acuerdo a los lineamientos del } \\
\text { Sistema de Gestión "H" } \\
\text { basándose en lo establecidos por } \\
\text { la norma Mexicana vigente NMX- }\end{array}$ \\
\hline
\end{tabular}

\begin{tabular}{|l|l|}
\hline & $\begin{array}{l}\text { F-NORMEX-2016 y la lista de } \\
\text { verificación. }\end{array}$ \\
\hline Paso 5. Validación & $\begin{array}{l}\text { Validación por parte del consultor } \\
\text { que el establecimiento cumpla con } \\
\text { lo necesario para posteriormente } \\
\text { solicitar la verificación y obtener la } \\
\text { certificación correspondiente. }\end{array}$ \\
\hline $\begin{array}{l}\text { Paso } 6 . \\
\text { Verificación }\end{array}$ & $\begin{array}{l}\text { El establecimiento contrata a la } \\
\text { Unidad de Verificación } \\
\text { (acreditadas por EMA-SECTUR) } \\
\text { de acuerdo con sus necesidades, } \\
\text { la cual llevará acabo la visita de } \\
\text { verificación final. }\end{array}$ \\
\hline $\begin{array}{l}\text { Paso 7. Emisión } \\
\text { del Distintivo "H" }\end{array}$ & $\begin{array}{l}\text { La Unidad de Verificación notifica } \\
\text { el resultado a la SECTUR, la cual } \\
\text { emite de manera oficial el } \\
\text { Distintivo "H". }\end{array}$ \\
\hline
\end{tabular}

Fuente: Adaptación por Ortega Ibarra, E., \& Hernández Jiménez, A. (2019). Manejo higiénico en el servicio de alimentos: Codex Alimentarius y Normas Oficiales Mexicanas.

Existen diversas normas, las cuales deben ser estudiadas a fondo por los estudiantes de la Licenciatura en Nutrición y áreas afines. No obstante, el conocimiento del área de Administración en Servicio a Colectividades debe considerar a las instituciones particulares y públicas, ya que de esta manera se podrá encasillar la NOM y la NMX para vigilar el cumplimiento. Sabiendo que existen además, diversidad de tipos de servicio al comensal, como el denominado "servicio a la mesa" de tipo "francés" (de etiqueta, el alimento es cocinado o completado junto a la mesa del comensal), "ruso" (estilo ceremonial, elegante, la atención es particular por parte del mesero, el comensal elige la cantidad que quiere ingerir), "inglés" (el mesero lleva a una mesa todos los alimentos, el comensal elige los alimentos y el mesero proporciona raciones delimitadas), "americano" (simple, informal; el mesero respeta hemisferios de servir y retirar trastos), "servicio de banquetes" (una misma comida por tiempos a todos los que estén en una mesa, cronometrado en ocasiones), "servicio de barra" (cafeterías, bares, hoteles, comedores industriales, el mesero está siempre detrás de una barra), "servicio buffet" (popular, cuanta por módulos, por tiempos, horarios, implica delimitar nombre de servicio (desayuno, almuerzo, cena), las porciones la decide el comensal), "servicio de cafetería" (no se venden bebidas alcohólicas que no se apeguen al café, son medidas y delimitadas, los alimentos son porciones para compartir). ${ }^{24}$ Estos tipos de servicios son delimitados por las instituciones, dependiendo el "giro comercial" o la "razón social". Tanto puede ser un hospital público, como un bar exclusivo. 
Hay que considerar que las normativas deben ser acatadas por las instituciones, independientemente de su tamaño, localización, razón social, giro comercial, clasificación ante organismo nacional que lo autoriza como la Cofepris. La vigilancia del cumplimiento es recurrente, más en unos que en otros, por el tipo de alimentos. Existe una diversidad extensa de lugares que ofrecen un solo alimento, hasta los que producen varios de esa misma materia prima. El control sanitario debe verse desde un punto de vista crítico, desde que el comensal es atendido, hasta cuando ingiere el producto final. Afortunadamente el nutriólogo ya es partícipe de instituciones tanto para vigilar, como para ser autoridad para emitir fallos, todo para asegurar la alimentación e idealmente la nutrición.

\section{Conclusión}

La Administración de Servicios de Alimentos, bajo la concepción funcional del proceso administrativo toma en cuenta las demandas actuales del campo laboral sobre innovación tecnológica en alimentos y servicios de alimentos, enmarcados por las teorías contemporáneas de sistemas y calidad, es decir que el nutriólogo deberá considerar las normativas institucionales, nacionales e internacionales, diferentes tipos de planeaciones (estratégicas y de contingencia), generalidades de procesos clave, de apoyo y control, interpretación de análisis microbiológicos, identificación de tipos de servicios al consumidor-comensal-usuario, planeación de menús, medición de satisfacción de quien ingiere el alimento, costos, gastos y control del almacén, compras y selección de proveedores, entre otros temas, de manera continua. La preocupación por mantener una cadena alimentaria bien controlada está ligada al deseo de obtener una alimentación de la máxima calidad y seguridad y esta relación entre calidad e inocuidad es de suma importancia debido a que es por ella que podemos prevenir las Enfermedades Trasmitidas por los Alimentos (ETA) que ya son un problema de Salud Pública.

De allí, la importancia de la Administración de Servicios de Alimentos ya que las actividades deben ser conducidas racionalmente y tener una buena base técnico-administrativa, donde el profesional de la Nutrición es el encargado de asumir e interactuar con el equipo de trabajo, respondiendo a las necesidades externas, internas y de alianzas estratégicas que surgen en la organización, mediante la aplicación de procesos administrativos y la elección adecuada de técnicas gerenciales. Desafortunadamente, hace falta que esta área de estudio de la nutriología sea mejor desarrollada, y publicada, ya que las referencias bibliográficas son limitadas; no obstante, se pueden solicitar las evaluaciones a las instituciones, pero implicaría que además hay que visualizar primero los procesos, métodos y técnicas para poder entender los porcentajes que se emiten por certificaciones oficiales nacionales 0 internacionales.

Aunado a esta escasez de referencias hay que dilucidar que en algunos tópicos que son presentados en estas mismas fuentes de datos nacionales e internacionales no se focalizan en administración de servicios de alimentación a colectividades de manera directa dado que la información que se presenta es somera y eso conlleva a que el docente obtenga información de distintas fuentes para tener una idea global de lo que implica esta temática. En la mayor parte de ocasiones los manuales que son realizados por instituciones sólo se centran en ciertos servicios de alimentación como comedores industriales, hoteles, restaurantes y hospitales, dejando por un lado ciertos servicios de alimentos que también son relevantes para el campo laboral del nutriólogo.

Lo anterior es solo un preámbulo de lo que implica el estudio de los Servicios de Alimentación, desde lo esencial, para proseguir y ser muy específicos en la institución, se espera que la sociedad científica mire a este rubro, porque diario lo convivimos, y en ocasiones se desconoce el fundamento teórico, administrativo y práctico.

\section{Referencias}

[1] Calvo Carrillo M de la C. Toxicología de los alimentos. Ciudad de México: McGraw-Hill; 2014.

[2] FAO. Normas internacionales [Internet]. 2015 [cited 2021 Feb 18]. Available from: http://www.fao.org/3/am859s/am859s14.pdf

[3] FAO. Informe de la cuadragésima primera reunión del comité del codex sobre higiene de los alimentos [Internet]. 2009 [cited 2021 Feb 18]. Available from: http://www.fao.org/tempref/codex/Reports/Alinorm10/al33_13s.pdf

[4] FAO/OMS. Codex Alimentarius - Higiene de los Alimentos (Textos básicos) - Cuarta edición [Internet]. 2009 [cited 2021 Feb 18]. Available from: http://www.fao.org/3/a1552s/a1552s00.htm

[5] Izquierdo Hernández A, Armenteros Borrell M, Lancés Cotilla L, Martín González I. Alimentación saludable. Rev Cuba enferm [Internet]. 2004 [cited 2021 Feb 18]; Available from: http://bvs.sld.cu/revistas/enf/vol20_1_04/enf12104.htm

[6] Guerrero Ramo CI. Administración de alimentos a colectividades y servicios de salud. In México: McGraw-Hill; 2001. p. 79-81.

[7] Mercado Zepeda, M. A., Lagos Sánchez, A. N., López Torres, L. P., \& Moreno Mejía LG. Administración de servicios de alimentos- Manual de prácticas. Editorial Universitaria. Universidad de Guadalajara; 2018. 
[8] Lopez P, Luz M, HUNOT C, Ortiz M, Aguayo M. La enseñanza de la gestión en los servicios de alimentos. Rev Educ y Desarro. 2011;16:6978.

[9] Dardano Sosa C. Lineamientos para organizar el servicio de nutricion y alimentacion. Costa Rica: Caja Costarricense del Seguro Social; 1985. $144 \mathrm{p}$.

[10] Tejada BD. Administración de servicios de alimentación: calidad, nutrición, productividad y beneficios. Editorial Universidad de Antioquia CS y D, editor. Medellín; 2007.

[11] Tejada BD. Administración de servicios de alimentación y nutrición. Perspect en Nutr Humana [Internet]. 2016 Jul 28 [cited 2021 Feb 19];17(2):195-6. Available from: https://revistas.udea.edu.co/index.php/nutricion/article/view/324669

[12] INN. Asesoría en Servicios de Alimentación [Internet]. 2020. Available from: https://www.inn.gob.ve

[13] Sylvia AB, RoseAnn M, John BK. Hygiene practices and food contamination in managed food service facilities in Uganda. African J Food Sci. 2015;9(1):31-42.

[14] Uyttendaele M, Franz E, Schlüter O. Food safety, a global challenge [Internet]. Vol. 13, International Journal of Environmental Research and Public Health. MDPI AG; 2015 [cited 2021 Feb 19]. Available from: /pmc/articles/PMC4730458/

[15] Ortega Ibarra E, Hernández Jiménez A. Manejo higiénico en el servicio de alimentos: Codex Alimentarius y Normas Oficiales Mexicanas. UVserva [Internet]. 2018 Oct 25 [cited 2021 Feb 19];0(6). Available from: https://uvserva.uv.mx/index.php/Uvserva/article/view/2584

[16] Intedya. NORMA OFICIAL MEXICANA 251 [Internet]. 2015. Available from: http://www.intedya.com/internacional/92/consultorianom-251-mexico.html

[17] Hellriegel D. Administración: un enfoque basado en competencias. 11th ed. Mexico: CENGAGE Learning; 2005. 39 \& 286 p.

[18] SSA. NORMA Oficial Mexicana NOM-251-SSA1-2009, Prácticas de higiene para el proceso de alimentos, bebidas o suplementos alimenticios [Internet]. 2009 [cited 2021 Feb 19]. Available from: https://www.dof.gob.mx/normasOficiales/3980/salud/salud.htm

[19] SSA. Norma Oficial Mexicana NOM-120-SSA1-1994 [Internet]. 1994 [cited 2021 Feb 23]. Available from: https://www.gob.mx/sfp/documentos/norma-oficial-mexicana-nom120-ssa1-1994

[20] SSA. NOM-093-SSA1-1994 [Internet]. 1994 [cited 2021 Feb 23]. Available from: http://www.salud.gob.mx/unidades/cdi/nom/093ssa14.html

[21] NORMA MEXICANA IMNC. Sistemas de gestión de calidadFundamentos y vocabulario, ISO 900:2000, COPANT/ISO 900-200, MXN-CC-9000-IMNC-2000. Diario Oficiald de la Federación. 2001.

[22] Martínez Ramírez A. Manual de gestión y mejora de procesos en los servicios de salud. Moderno M, editor. México; 2005.

[23] SECTUR. Programa Manejo Higiénico de los Alimentos, Distintivo "H"| Distintivo H [Internet]. 2014 [cited 2021 Feb 20]. Available from: http://www.calidad.sectur.gob.mx/distintivoh/2014/10/17/programamanejo-higienico-de-los-alimentos-distintivo-h/

[24] Dahmer S. Restaurantes: servicio básico. Acribia, editor. España; 2002. 13-30 p. 